kurzen Stößen. Die Spannung steigt in jedem StoB schnell auf ihren Maximalwert, bleibt dort eine kurze Zeit konstant und fällt dann wieder schnell auf Null.

Betrachtet man jeden Spannungsstoß einzeln, so kann man jetzt annehmen, daß der Verlauf der Spannung an der Röhre ebenso verläuft, wie wenn man beim Betriebe mit kontinuierlichem Gleichstrom den Strom offnet, einen kurzen Augenblick schließt und wieder öffnet. Bei den schnellen Anderungen am Beginn und Ende eines jeden StoBes wird die Hysteresis zur Wirkung kommen, und zwar so, daß am. Anfang des Stobes die Spannungskurve einen hohen Zündgipfel überschreiten muB, und daß am Ende die Leitfähigkeit in der Röhre noch so lange erhalten bleibt, daß die Spannung ohne weiteres auf Null herabfällt. Bisher ist jeder StoB einzeln betrachtet und angenommen, daB bei ihm die Schnelligkeit der Bildung und Wiedervereinigung der Tonen im Innern der Röhre eine Rolle spielt. Dabei sollte sich die Temperatur der Röhre auf einen Mittelweri einstellen und keine wesentlichen Schwankungen aufweisen. Fs ist nun nicht zu verkenmen, dab die Temperatursehwankungen der Röhre beim praktischen Betriebe dennoch eine Rolle spielen können, und zwar besonders dann, wenn die Pausen zwischen den StöBen ziemlich lang sind. Es wird so der Fall eintreten können, daß die Zündispannung beim Beginn jedes Stoßes nicht mehr den von $H$. Kröncke gefundenen Wert hat, sondern dab die Röhre in der dazwischenliegenden Pause Zeit gehabt hat, sich abzukühlen, und daß daher die Zündspannung höher liegt. Mit abnehmender Stoßzahl wird daher die Zündspannung der Röhre steigen. Zugleich folgt daraus, daß mit abnehmender Stoßzahl die Härte einer Röhre größer wer. den wird.

Mit Hilfe der neuen Theorie ist es dem Verfasser möglich, eine große Anzahl von Erscheinungen, die man im praktischen Röntgenbetriebe beobachtet hat, zu erklären und zugleich einen umfassenden Einblick in das Gebiet zu gewähren. Zum Schlus wird ein wichtiges Problem der Röntgentechnik einer neuen Lösung zugeführt. Bekanntlich hat man sich in der letzten Zeit die Aufgabe gestellt, die Röntgenstrahlen so hart zu machen, daß sie in der Tiefentherapie in erfolgreichen Wettbewerb mit den $\gamma$-Strahlen des Ra diums treten können. Würde dies gelingen, so würde es möglich sein, an Stelle der sehr kostspieligen Radiumpräparate die viel billiger zu betreibende Röntgenröhre zu setzen, wenigstens soweit es sich um die Bebandlung mit durchdringungsfähigen Strahlen handelt. Der neue Vorschlag des. Verfassers geht dahin, nur aurch die Zündspannung in der Röhre Röntgen. strahlen zu erzeugen. Dies soll dadurch erreicht wer den, daß vor die Röbre ein großer Vorschaltwiderstand geschaltet wird, um den stabilen Betriebszustand möglichst auf den abfallenden Ast der Charakteristik zu verlegen. Ferner werden in den Röntgenröhrenstromkreis noch Luftiunkenstrecken eingeschaltet, welche die Wirkung haben, den Stromdurchgang abzukürzen. Bei dieser neuen Betriebsform würde durch die Röntgenröhre eine Entladung gehen, die einer Funkenladung - im Gegensatz zu der lichtbogenähnlichen Entladung bei gewöhnlichem Betrieb - entspricht.

Ein neues Röntgenrohr für spektroskopisehe Zweeke. (Siegbahn, Verhandlungen der Deutschen Physikalischen Gesellschaft, Bd. 17, S. 469, 1915.) Bei der röntgenspektroskopischen Untersuchung der verschiedenen Elemente fertigt man im allgemeinen die Antikathode der Röntgenröhre aus dem zu untersuchenden Filement an oder man läßt die von einer beliebigen Antikathode ausgehenden Röntgenstrahlen auf ein Stuck des zu untersuchenden Elementes fallen und dort sekundäre Röntgenstrahlen erzeugen, die für das Element charakteristisch sind, und untersucht diese dann mit einer der ublichen Kristallinterferenzmethoden. Die erste Methode läßt sich dann nicht verwen. den, wenn die Substanz das für das Röhreninnere erforderliche Vakuum nicht vertragen kann, also z. B. sehr leicht verdampft; bei der zweiten Methode treten dadurch Schwierigkeiten auf, daß die Intensität der Sekundärstrahlen sehr gering ist und daß man daher bei der spektroskopischen Aufnahme zu sehr langen Belichtungszeiten gelangt. Vom Verfasser wird eine Röntgenröhre angegeben, die für spektroskopische Aufnahmen nach der zweiten Methode geejgnet ist. Die Röhre ist ähnlich wie die von Zehnder angegebene aus Metall hergestellt, das durch Kiihlrippen gekïhlt wird, und in das mittels eines. Porzellanisolators die Kathode eingefuhrt ist. Die Antikathode befindet sich in der Wandung der Röhre und besteht ans einem $0,15 \mathrm{~mm}$ dicken Silberblech. Direkt an diese Antikathode wird das zu untersuchende Element auBen angelegt und in ihm daher eine intensive Selkundär strahlung erzeugt. Während des Betriebes bleibt die mit Pizein gedichtete Röhre in Verbindung mit einer Molekularpumpe. Es ist so möglich, eine große Anzahl von Elementen röntgenspektroskopisch zu untersuchen. $\quad$ P. Ludewig, Freiberg i. Sa.

\section{Technische Mitteilungen}

Den sehwarzen Phosphor, der bei $200^{\circ}$ unter $12000 \mathrm{~kg}$ Druck aus weißem Phosphor entsteht, hat $P$. W. Bridgman auch aus rotem Phosphor herzustellen versucht. Der rote Phosphor ist kein einheitlich bestimmter Stoff, sondern verschieden in Aussehen und Dichte je nach dem Herstellungsverfahren. Die Arten, welche die gröBte Dichte besitzen, haben eine violette Färbung. Bei den Versuchen wurden zwei verschiedene Arten benutzt, eine Probe hellroten Phosphors, die bei $500 \mathrm{~kg}$ Druck in einer Stickstoffatmosphäre gebildet war, und eine Probe violetten Phosphors. Der rote Phosphor wurde mit weiBem Phosphor amgeben und die ganze Masse bei $200^{\circ}$ einem Druck von $12500 \mathrm{~kg}$ ausgesetzt. Dadurch wurde der weiße Phosphor in schwarzen Phosphor verwandelt, der rote blieb ganz unverändert mitten in der Masse des schwarzen Phosphors. Sodann wurde weißer Phosphor, für dessen Verwandlung in roten Phosphor Natriummetall als Katalysator dient, mit einer Spur Natrium bei Zimmertemperatur auf einen Druck von $4000 \mathrm{~kg}$ gebracht und bei konstantem Volumen auf $200^{\circ}$ erhitzt. Dadurch stieg sein Druek um $500 \mathrm{~kg}$, der bei $200^{\circ}$ auf $12500 \mathrm{~kg}$ erhöht und nach 20 Minuten auf $13000 \mathrm{~kg}$ gesteigert wurde. Nach der Abkühlung war der Phosphor in seiner ganzen Masse violett und die erwartete Umwandlung in schwarzen Phosphor vollständig ausgeblieben. Auch der Versuch, Jod als Katalysator für diese Umwandlung zu benutzen, miBlang und ebenso alle sonstigen Versuche, sehwarzen Phosphor aus rotem oder violettem herzustellen. Die Ergebnisse seiner Versuche stellt Verf. in folgenden drei Sätzen dar: 1. Schwarzer Phosphor ist stabil gegen weiken bei $200^{\circ}$ und unter Drucken, die größer sind als $4000 \mathrm{~kg}$. 2. Violetter Phosphor ist stabil gegen weißen bei $4000 \mathrm{~kg}$ Druck und in Tempe- 
raturen etwas unterhalb $200^{\circ}$. 3. Violetter Phosphor ist stabil gegen roten bei Drucken zwischen 8000 und $12000 \mathrm{~kg}$. (Journ. Am. Chem. Soc. 38, 609, 1916.)

Ùber die Herstellung von Kobaltniederschlägen auf anderen Metallen und Legierungen, wie Messing, Eisen, Stahl, Kupfer, Zinn, Neusilber, Blei und Britanniametall, baben H. T. Kalmus, C. H. Harper und W. L. Savell sehr urnfangreiche Versuche angestellt. Aus der großen Zahl der erprobten Bäder empfehlen sie folgende zwei Lösungen: Lösung A, $200 \mathrm{~g}$ kristallisier. tes Kobaltammoniumsulfat $\left(\mathrm{CoSO}_{4}\left(\mathrm{NH}_{4}\right)_{2} \mathrm{SO}_{4} .6 \mathrm{H}_{2} \mathrm{O}\right)$ auf 11 Wasser und Lösung B, $312 \mathrm{~g}$ Kobaltsulfat $\left(\mathrm{CoSO}_{4}\right)+$ $19,6 \mathrm{~g}$ Kochsalz + Borsäure bis zur Sättigung auf 1 l Wasser. Die mit diesen Lösungen herzustellenden Niedersebläge sind dicht, fest, anhaftend, hart und gleichmäBig. Sie sind sehr leicht zu einer Oberfläche von vollkommen befriedigender Glätte zn polieren, die eine glänzend weiBe Farbe mit einem schwach bläulichen Schimmer besitzt. Dabei haben diese Niederschläge vor Nickelniederschlägen bei ihrer Berstellung eine große Reihe von Vorzugen. Die elektrische Leitfähigkeit der Lösungen ist größfer als bei den gebräuchlichen Nickelbädern, so daß die Kobaltbäder einer geringeren Spannung bedürfen. Besonders hervortretend ist die Schnelligkeit, mit der sich die Kobaltnieder. schläge herstellen lassen. Lösung A liefert die Nieder. schläge 4 mal schneller als die am geschwindesten ar beitenden Nickelbäder und bei Lösung $B$ ist die Geschwindigkeit sogar 15 mal so groß. Die mit so großer Schnelligkeit erzeugten Niederschläge sind viel härter als Nickelniederschläge, sie können daher dünner sein und trotzdem den gleichen Sehutz für die Oberfläche leisten. Beim Arbeiten mit der Lösung B unter Ver. wendung von 16 Amp. auf 1 qdom erfordern Automobilteile, Messingstempel usw. für Vollendung des Kobaltuberzuges einschließlich der Politur nur $1 \mathrm{Mi}$ nute, die besten Nickelbäder dagegen beim Betriebe mit 1,1 Amp. auf 1 qdem 1 Stunde. Hiernach braucht $\operatorname{man} \mathrm{zu}$ den Kobaltniederschlägen nur $1 / 4$ so viel Metall wie zu den Nickelniederschlägen. Kobalt kann also 4-mal so tever sein wie Nickel, und dennoch ist seine Verwendung vorteilhafter, weil sie Arbeit und Zeit erspart. In einem kleinen Raum kann anch mehr Arbeit mit Kobalt-als mit Nickelbüdern geleistet werden. Kobaltüberzüge, die zum Schmuck auf Messing, Kupfer, Zink oder Neusilber angebracht werden, können in einer Minute hergestellt werden. Waren, die starker Schüdigung durch Wettereinflüsse oder durch Reibung ausgesetzt sind, erfordern hö̋hstens 15 Minuten. Die Russel Motor Car Company hat nit Kobaltuberzügen. auf Automobilteilen und Schlittschuhen sehr guinstige Erfahrungen gemacht, und bei den gegenwärtigen Zeitumständen. wird der Ersatz des Nickels durch Kobalt bei uns in Deutschland ganz besonders hoch zu schätzen sein. (Journ. of Ind. and Eng. Chem. 7, 379, 1915.)

Eine Haarsalbe aus der Zeit der alten Römer ist von $L$. Reutter analysiext worden, Bei Ausgrabungen, die in Lugano von dem Direktor des dortigen städtischen Museums, $M$. Bally, vorgenommen wurden, fand man einen alten römischen Krug, der eine ziemlich weiche, salbenartige, fettige Masse enthielt mit einem Geruche nach Terpentin und Storaxharz und von gelbbrauner Farbe. Beim Erwärmen schmolz sie gegen $58^{\circ} \mathrm{zu}$ einer gelblichen Flüsigkeit mit vielen mineralischen und pflanzlichen festen Bestandteilen. Die weitere Untersuchung zeigte, daß sie teilweise in $\mathbf{P e}$ troleumäther, Äther, Alkohol und Chloroform löslich war. Der in Petroleumäther gelöste Bestandteil ergab in der
Elementaranalyse die Formel $\mathrm{C}_{28} \mathrm{H}_{54} \mathrm{O}_{2}$. Dies deutet darauf, daß die Masse ein Gemisch ron Bienenwachs und Fetten darstellte, dem die Römer Storaxharz und Terpentin zugesetzt hatten. Die beiden letzten Bestandteile waren in Weinsäure eingeweicht worden. Außerdem war Henna der braunen Färbung und des Geruches wegen zugefügt, sowie ein Gerbstoff, der die Masse konservieren und ihr einen besonderen Duft verlethen sollte. Dies Gemenge diente in der Zeit der alten Römer den Modedamen als Salbe fur die Haut oder die Haare. (C. R. 162, 470, 1916.)

Einen die Benetzung verhindernden Überzug auf Sandkörnern und anf der Oberfläche der Ackererde glaubt $H$. Devaus annehmen zu könner. Bringt man nämlich trockenen Sand, dessen Körner durchschnittlich Durchmesser von 0,37 bis $0,67 \mathrm{~mm}$ besitzen, vor* sichtig auf die Oberfläche von Wasser, so bleiben 60-63 \% davon schwimmend. Wird er schwach angefeuchtet, so daB ex bis zu $1 / 2 \%$ Wasser enthält, so schwimmt er noch besser; bis zu $90 \%$ bleiben dann davon auf dem Wasser. Mit einem Gehalt von $1 \%$ Wasser oder mehr sinken aber alle Sandkörner sofort unter. Die Schwimmiähigkeit der Sandkörner beruht darauf, daß sie unvollständig benetzt sind. Ein kleiner Teil ihrer. Oberflïche bleibt trocken, selbst wenn sie tagelang auf dem Wasser treiben. Auch ganz untergetauchte Körner können, an die Oberfläche gebracht, wieder schwimmend werden. Die Ursache für die un. vollkommene Benetzung sieht Devaux in einer Hülle, welche die Sandkörner bedeckt und ähnlich wie Wachs wirkt, mit dem man eine Stahlnadel schwimmend machen kann. Wird Sand nämlich ausgeglüht, so verliert er seine Schwimmfähigkeit. Alle Körner sinken sofort unter und behalten diese Eigensehaft für mehrere Tage. Die Wärme hat somit eine organische Hülle, welche ihre Oberfläche wahrscheinlich bedeckte zerstört. Hierauf deutet auch folgender Versuch: Wird eine Wasseroberfläche in dünner Schicht mit einem unlöslichen Pulver, etwa Talk, bestreut, und läßt man dann Sandkörner darauf fallen, so treibt jedes Korn die Talkkörnchen weit von sich fort, was beweist, daß durch die Sandkörner auf dem Wasser ein fremder Stoff von schwacher Oberflächenspánnung zuräekgelassen ist. Die gleichen Erscheinungen zeigt auch Ackererde. Das Vorhandensein einer solchen Hiulle muB notwendigerweise eine bedeutende Einwirkung anf die kapillaren Eigenschaften dieses für die Pflanzenwelt so wichtigen Stoffes ausüben. (C.R. 162, 197, 1916, )

Briketts in einer fiur die Sehiffahrt brauchbaren Form herzustellen ist $D$. A. Thomas und $H$.J. Phillipps gelungen. Bisher wurden die Briketts mit Hilfe von Bindemitteln wie Teer, Glukose, Melasse usw. geprent. Dies hatte zur Folge, daB sie bei der Verbrennung viel Rauch erzeugten und deswegen auf Schiffen nicht verwandt werden konnten. In England wurden daher jährilich nur kaum 2 Millionen Tonnen Briketts hergestellt, während die Erzeugung in Dentschland mehr als 20 Millionen betrug und auch in Frankreich sehr bedeutend war. Nach dem neuen Verfahren werden die Briketts ohne jedes Bindemittel unter sehr hohem Druck aus gepulverten Kohlen geprebt. Die so hergestellten Kohleblöcke sind luftbeständig und geben nieht mehr Ranch als gewöhnliche Kohlen. Nachdem dies Verfahren 1 bis 2 Jahre erprobt ist, wird es nunmehr in Süd-Wales in großem Maßstabe angewandt. (Journ. Ind. and Eng. Chem. 7, 544, 1915.)

A. Mahlke, Hamburg. 